\title{
Prevalence of Prediabetes and Undiagnosed Diabetes in Patients with HFpEF and HFrEF and Associated Clinical Outcomes
}

\author{
Søren L. Kristensen ${ }^{1,2}$ - Pardeep S. Jhund ${ }^{1}$ Matthew M. Y. Lee ${ }^{1}$ Lars Køber $^{2}$ • \\ Scott D. Solomon ${ }^{3}$. Christopher B. Granger ${ }^{4} \cdot$ Salim Yusuf ${ }^{5}$ - Marc A. Pfeffer ${ }^{3}$. \\ Karl Swedberg ${ }^{6,7}$ • John J. V. McMurray ${ }^{1}$ - CHARM Investigators and Committees
}

Published online: 25 September 2017

(C) The Author(s) 2017. This article is an open access publication

\begin{abstract}
Purpose The prevalence and consequences of prediabetic dysglycemia and undiagnosed diabetes is unknown in patients with heart failure (HF) and preserved ejection fraction (HFpEF) and has not been compared to heart failure and reduced ejection fraction (HFrEF).

Methods We examined the prevalence and outcomes associated with normoglycemia, prediabetic dysglycemia and diabetes (diagnosed and undiagnosed) among individuals with a baseline glycated hemoglobin (hemoglobin $\mathrm{Alc}, \mathrm{HbA1c}$ ) measurement stratified by HFrEF or HFpEF in the Candesartan in Heart failure Assessment of Reduction in Mortality and morbidity programme (CHARM). We studied the primary outcome of HF hospitalization or cardiovascular $(\mathrm{CV})$ death, and all-cause death, and estimated hazard ratios (HR) by use of multivariable Cox regression models.
\end{abstract}

John J. V. McMurray

john.mcmurray@glasgow.ac.uk

1 British Heart Foundation Cardiovascular Research Centre, Institute of Cardiovascular and Medial Sciences, University of Glasgow, 126 University Place, Glasgow G12 8TA, UK

2 Department of Cardiology, Rigshospitalet University Hospital, Copenhagen, Denmark

3 Division of Cardiovascular Medicine, Brigham and Women's Hospital, Boston, MA, USA

4 Duke Clinical Research Institute, Duke University Medical Center, Durham, NC, USA

5 Population Health Research Institute, McMaster University, Hamilton, ON, Canada

6 Department of Molecular and Clinical Medicine, Sahlgrenska Academy, University of Gothenburg, Göteborg, Sweden

7 National Heart and Lung Institute, Imperial College, London, UK
Results $\mathrm{HbA1c}$ was measured at baseline in CHARM patients enrolled in the USA and Canada and was available in 1072/3023 (35\%) of patients with HFpEF and 1578/4576 (34\%) patients with HFrEF. 18 and $16 \%$ had normoglycemia $(\mathrm{HbAlc}<6.0), 20$ and $22 \%$ had prediabetes (HbA1c 6.0-6.4), respectively. Finally among patients with HFpEF $22 \%$ had undiagnosed diabetes ( $\mathrm{HbAlc}>6.4$ ), and $40 \%$ had known diabetes (any $\mathrm{HbAlc}$ ), with corresponding prevalence among HFrEF patients being 26 and $35 \%$. The rates of both clinical outcomes of interest were higher in patients with undiagnosed diabetes and prediabetes, compared to normoglycemic patients, irrespective of HF subtype, and in general higher among HFrEF patients. For the primary composite outcome among HFpEF patients, the HRs were 1.02 (95\% CI 0.63-1.65) for prediabetes, HR $1.18(0.75-1.86)$ for undiagnosed diabetes and 2.75 (1.83-4.11) for known diabetes, respectively, $p$ value for trend across groups $<0.001$. Dysglycemia was also associated with worse outcomes in HFrEF.

Conclusions These findings confirm the remarkably high prevalence of dysglycemia in heart failure irrespective of ejection fraction phenotype, and demonstrate that dysglycemia is associated with a higher risk of adverse clinical outcomes, even before the diagnosis of diabetes and institution of glucose lowering therapy in patients with $\mathrm{HFpEF}$ as well as HFrEF.

Keywords Heart failure $\cdot$ Heart failure and preserved ejection fraction $\cdot$ Diabetes $\cdot$ Dysglycemia $\cdot$ Prognosis

\section{Introduction}

People with diabetes have a higher risk of developing incident heart failure (HF) than those without diabetes [1-3] and among patients with $\mathrm{HF}$, those with diabetes have worse outcomes than patients without diabetes [4-6]. The latter observation is of considerable import because the prevalence of co-existing 
diabetes is high among patients with HF, affecting between 25 and $50 \%$ of individuals depending on which study is examined.

Recently, both undiagnosed diabetes and prediabetic dysglycemia were also found to be common in patients with $\mathrm{HF}$ with reduced ejection fraction (HFrEF) and each was associated with worse outcomes, compared with normoglycemia, although the risk was not as high as in patients with diagnosed diabetes [7]. The aim of our study was to determine the prevalence and prognostic significance of undiagnosed diabetes and prediabetic dysglycemia in the other major HF phenotype, HF with preserved ejection fraction (HFpEF), and compare the prevalence and outcomes with those in contemporaneously recruited patients with HFrEF. To do this, we used data from the Candesartan in Heart failure: Assessment of Reduction in Mortality and morbidity (CHARM) programme, which included patients with HFrEF and HFpEF [6, 8].

\section{Methods}

The CHARM-Programme consisted of one HFpEF trial and two HFrEF trials. CHARM-Preserved enrolled 3023 patients
18 years or older, in York Heart Association (NYHA) functional class II-IV, with a prior hospitalization for a cardiac reason and a left ventricular ejection fraction (LVEF) above $40 \%$ [8]. CHARM-Alternative and CHARM-Added included 2028 and 2548 patients, respectively, aged 18 years or older, in NYHA functional class II-IV, with a LVEF of $40 \%$ or less, either treated with an angiotensin converting enzyme (ACE) inhibitor (CHARM-Added) or not, because of intolerance (CHARM-Alternative) [8]. As previously reported, glycated hemoglobin (hemoglobin A1c, HbA1c) was measured at baseline in the 2650 CHARM patients enrolled in Canada and the USA [6]. We categorized glycemic status in individuals without a history of diabetes using the International Diabetes Expert Committee criteria: normoglycemia (HbA1c < 6.0\%), prediabetic dysglycemia (HbA1c 6.0$6.4 \%$ ) and undiagnosed diabetes (HbAlc > 6.4\%) [9]. Patients with a prior diagnosis of diabetes were considered to have diabetes irrespective of HbAlc level.

We used multivariable Cox proportional hazards models to evaluate the primary composite outcome of CHARM, which was death from cardiovascular (CV) causes or a hospitalization for HF, as well as all-cause mortality, according to glycemic
Table 1 Baseline characteristics of patients with in CHARMPreserved with $\mathrm{HbA1c}$ available, according to glycemic status

\begin{tabular}{|c|c|c|c|c|c|}
\hline & \multicolumn{3}{|c|}{ No prior diagnosis of diabetes } & \multirow{2}{*}{$\begin{array}{l}\text { Prior diabetes } \\
\text { Any HbA1c }\end{array}$} & \multirow{2}{*}{$p$ value } \\
\hline & $\mathrm{HbA} 1 \mathrm{c}<6.0$ & HbA1c $6.0-6.4$ & $\mathrm{HbA} 1 \mathrm{c}>6.4$ & & \\
\hline Patients, no (\%) & $189(18 \%)$ & $217(20 \%)$ & $238(22 \%)$ & $428(40 \%)$ & \\
\hline Age, years & $63 \pm 12$ & $67 \pm 11$ & $69 \pm 11$ & $65 \pm 11$ & $<0.001$ \\
\hline Female, $n(\%)$ & $82(43 \%)$ & $94(43 \%)$ & $100(42 \%)$ & $187(44 \%)$ & 0.98 \\
\hline HbA1c, median (Q1-Q3) & $5.6(5.5-5.7)$ & $6.1(6.0-6.2)$ & $6.7(6.5-7.1)$ & $7.8(7.1-9.0)$ & $<0.001$ \\
\hline NYHA class, $n(\%)$ & & & & & 0.0006 \\
\hline II & $105(56 \%)$ & $120(55 \%)$ & $115(48 \%)$ & $168(39 \%)$ & \\
\hline III & $81(43 \%)$ & $94(43 \%)$ & $119(50 \%)$ & $246(57 \%)$ & \\
\hline IV & $3(2 \%)$ & $3(1 \%)$ & $4(2 \%)$ & $14(3 \%)$ & \\
\hline Ejection fraction & $0.56 \pm 0.10$ & $0.55 \pm 0.09$ & $0.55 \pm 0.09$ & $0.54 \pm 0.09$ & 0.25 \\
\hline Heart rate, bpm & $68 \pm 11$ & $69 \pm 11$ & $70 \pm 12$ & $72 \pm 11$ & $<0.001$ \\
\hline SBP, $\mathrm{mmHg}$ & $132 \pm 19$ & $133 \pm 17$ & $133 \pm 18$ & $134 \pm 17$ & 0.86 \\
\hline BMI, $\mathrm{kg} / \mathrm{m}^{2}$ & $29.4 \pm 6.4$ & $29.8 \pm 6.3$ & $29.8 \pm 6.8$ & $32.9 \pm 7.1$ & $<0.001$ \\
\hline eGFR, $\mathrm{ml} / \mathrm{min} / 1.73 \mathrm{~m}^{2}$ & $82 \pm 22$ & $74 \pm 23$ & $70 \pm 25$ & $69 \pm 27$ & $<0.001$ \\
\hline \multicolumn{6}{|l|}{ Medical history, $n(\%)$} \\
\hline Ischemic etiology & $81(43 \%)$ & $108(50 \%)$ & $122(51 \%)$ & $241(56 \%)$ & $<0.001$ \\
\hline Prior $\mathrm{CABG}$ & $39(21 \%)$ & $59(27 \%)$ & $67(28 \%)$ & $144(34 \%)$ & $<0.001$ \\
\hline Prior PCI & $33(17 \%)$ & $43(20 \%)$ & $52(22 \%)$ & $94(22 \%)$ & 0.59 \\
\hline Prior stroke & $16(8 \%)$ & $24(11 \%)$ & $19(8 \%)$ & $53(12 \%)$ & 0.25 \\
\hline Prior AF & $51(27 \%)$ & $73(34 \%)$ & $87(37 \%)$ & $121(28 \%)$ & 0.07 \\
\hline Loop diuretic & $110(58 \%)$ & $144(66 \%)$ & $162(68 \%)$ & $319(75 \%)$ & $<0.001$ \\
\hline Digoxin & $56(30 \%)$ & $68(31 \%)$ & $87(37 \%)$ & $158(37 \%)$ & 0.21 \\
\hline$\beta$-blocker & $106(56 \%)$ & $123(57 \%)$ & $129(54 \%)$ & $243(57 \%)$ & 0.93 \\
\hline MRA & $17(9 \%)$ & $22(10 \%)$ & $18(8 \%)$ & $52(12 \%)$ & 0.27 \\
\hline
\end{tabular}

$H b A 1 c$ hemoglobin A1c, NYHA New York Heart Association functional class, SBP systolic blood pressure, $B M I$ body mass index, $e G F R$ estimated glomerular filtration rate, $C A B G$ coronary artery bypass graft, $P C I$ percutaneous coronary intervention, $A F$ atrial fibrillation, $M R A$ mineralocorticoid receptor antagonist 
status. Analyses were adjusted for age, sex, treatment arm, ejection fraction, NYHA class, heart rate, systolic blood pressure, body mass index (BMI), history of coronary artery bypass grafting, percutaneous coronary intervention, implantable cardioverter defibrillator, stroke and atrial fibrillation.

\section{Results}

HbAlc was available in 1072/3023 (35\%) of the patients in CHARM-Preserved and 428 patients with a HbA1c measurement in this trial had a history of diabetes (Table 1). Patients with diabetes were older, had a higher BMI, more evidence of coronary heart disease, worse NYHA class, higher heart rate, lower estimated glomerular filtration rate (eGFR) and greater use of loop diuretics compared to those with normoglycemia. Patients with previously undiagnosed diabetes and those with prediabetes had a clinical picture in between individuals with known diabetes and those with normoglycemia (Table 1).

HbA1c was available in 1578/4576 (34\%) of individuals in CHARM-Alternative and CHARM-Added, and 558 patients in these trials with a HbAlc measurement had a history of diabetes (Table 2). Similar to what was observed in HFpEF, we found that patients with known or undiagnosed diabetes were older, had a worse NYHA class distribution and kidney function and were more likely to have evidence of coronary heart disease.

Only $18 \%$ of patients with HFpEF and $16 \%$ of patients with HFrEF were normoglycemic.

Prediabetes was more common than normoglycemia in both types of HF: $20 \%$ in patients with HFpEF and $22 \%$ in those with HFrEF $(p=0.25)$.

The prevalence of undiagnosed diabetes was also high, but was less common in patients with HFpEF compared with HFrEF (22 vs. $26 \%, p=0.01$ ). Conversely, the prevalence of known diabetes was higher in patients with HFpEF (40 vs 35\%, $p=0.02$ ). As a result, the prevalence of any diabetes (diagnosed and previously undiagnosed) was $62 \%$ in each study.

$\mathrm{HFpEF}$ and HFrEF patients with diagnosed diabetes were at significantly higher risk of both the primary composite outcome, and all-cause mortality, compared with normoglycemic patients (Figs. 1 and 2). The rates of both outcomes of interest were
Table 2 Baseline characteristics of patients with in CHARMAdded/Alternative with $\mathrm{HbAlc}$ available, according to glycemic status

\begin{tabular}{|c|c|c|c|c|c|}
\hline & \multicolumn{3}{|c|}{ No prior diagnosis of diabetes } & \multirow{2}{*}{$\begin{array}{l}\text { Prior diabetes } \\
\text { Any HbAlc }\end{array}$} & \multirow[t]{2}{*}{$p$ value } \\
\hline & $\mathrm{HbA} 1 \mathrm{c}<6.0$ & $\mathrm{HbA1c} 6.0-6.4$ & $\mathrm{HbA1c}>6.4$ & & \\
\hline Patients, no (\%) & $254(16 \%)$ & $349(22 \%)$ & $417(26 \%)$ & $558(35 \%)$ & \\
\hline Age, years & $61 \pm 13$ & $65 \pm 12$ & $67 \pm 11$ & $64 \pm 10$ & $<0.001$ \\
\hline Female, $n(\%)$ & $67(26 \%)$ & $93(27 \%)$ & $108(26 \%)$ & $152(27 \%)$ & 0.97 \\
\hline HbA1c, median (Q1-Q3) & $5.6(5.4-5.7)$ & $6.1(6.0-6.2)$ & $6.7(6.5-7.1)$ & $8.1(7.1-9.3)$ & $<0.001$ \\
\hline NYHA class, $n(\%)$ & & & & & 0.028 \\
\hline II & $93(37 \%)$ & $104(30 \%)$ & $124(30 \%)$ & $139(25 \%)$ & \\
\hline III & $155(61 \%)$ & $238(68 \%)$ & $278(67 \%)$ & $398(71 \%)$ & \\
\hline IV & $6(2 \%)$ & $7(2 \%)$ & $15(4 \%)$ & $21(4 \%)$ & \\
\hline Ejection fraction & $0.28 \pm 0.08$ & $0.29 \pm 0.09$ & $0.27 \pm 0.08$ & $0.27 \pm 0.08$ & $<0.001$ \\
\hline Heart rate, bpm & $71 \pm 12$ & $72 \pm 12$ & $72 \pm 12$ & $75 \pm 12$ & $<0.001$ \\
\hline SBP, mmHg & $125 \pm 18$ & $124 \pm 19$ & $123 \pm 19$ & $126 \pm 19$ & 0.09 \\
\hline BMI, $\mathrm{kg} / \mathrm{m}^{2}$ & $27.9 \pm 4.9$ & $27.5 \pm 5.7$ & $28.1 \pm 5.7$ & $30.3 \pm 6.1$ & $<0.001$ \\
\hline $\mathrm{eGFR}, \mathrm{ml} / \mathrm{min} / 1.73 \mathrm{~m}^{2}$ & $81 \pm 23$ & $72 \pm 24$ & $68 \pm 25$ & $67 \pm 26$ & $<0.001$ \\
\hline \multicolumn{6}{|l|}{ Medical history, $n(\%)$} \\
\hline Ischemic etiology & $134(53 \%)$ & $212(61 \%)$ & $268(64 \%)$ & $390(70 \%)$ & $<0.001$ \\
\hline Prior CABG & $78(31 \%)$ & $112(32 \%)$ & $142(34 \%)$ & $216(39 \%)$ & 0.08 \\
\hline Prior PCI & $42(17 \%)$ & $57(16 \%)$ & $80(19 \%)$ & $137(25 \%)$ & 0.007 \\
\hline Prior stroke & $19(7 \%)$ & $31(9 \%)$ & $54(13 \%)$ & $63(11 \%)$ & 0.091 \\
\hline Prior AF & $62(24 \%)$ & $92(26 \%)$ & $128(31 \%)$ & $149(27 \%)$ & 0.29 \\
\hline Loop diuretic & $174(69 \%)$ & $258(74 \%)$ & $333(80 \%)$ & $460(82 \%)$ & $<0.001$ \\
\hline Digoxin & $165(65 \%)$ & $222(64 \%)$ & $267(64 \%)$ & $394(71 \%)$ & 0.07 \\
\hline$\beta$-blocker & $136(54 \%)$ & $163(47 \%)$ & $232(56 \%)$ & $330(59 \%)$ & 0.003 \\
\hline MRA & $44(17 \%)$ & $52(15 \%)$ & $93(22 \%)$ & $100(18 \%)$ & 0.06 \\
\hline
\end{tabular}

$H b A 1 c$ hemoglobin A1c, NYHA New York Heart Association functional class, SBP systolic blood pressure, BMI body mass index, $e G F R$ estimated glomerular filtration rate, $C A B G$ coronary artery bypass graft, $P C I$ percutaneous coronary intervention, $A F$ atrial fibrillation, $M R A$ mineralocorticoid receptor antagonist 
CV death or HFH No previous diagnosis of DM Normoglycemia (HbA1c<6.0) Prediabetes (HbA1c 6.0-6.4) Diabetes ( $\mathrm{HbA} 1 \mathrm{c}>6.4)$ Previous DM (any HbA1c)

All-cause mortality No previous diagnosis of DM Normoglycemia ( $\mathrm{HbA} 1 \mathrm{c}<6.0)$ Prediabetes (HbA1c 6.0-6.4) Diabetes (HbA1c >6.4) Previous DM (any HbA1c)

$\begin{gathered}\text { Number } \\ \text { events/ } \\ \text { patients }\end{gathered}$

$30 / 189$
$40 / 217$
$55 / 238$
$176 / 428$




$17 / 189$
$24 / 217$
$43 / 238$
$90 / 428$

\section{CHARM Preserved}

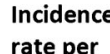

100 py

Adj. HR (95\% Cl)

1.00 (ref.)

$1.02(0.63-1.65)$

$1.18(0.75-1.86)$

$2.75(1.83-4.11)$

\section{7 \\ 6.9 \\ 8.8
18.5}

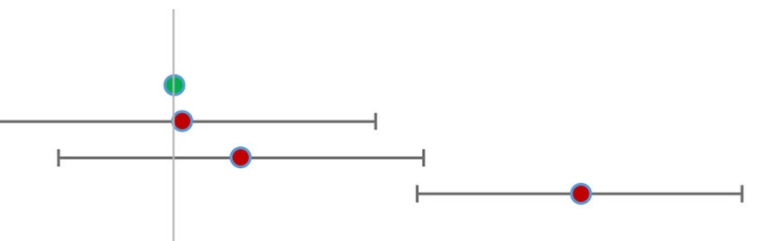

1.00 (ref.)

$1.06(0.57-1.99)$

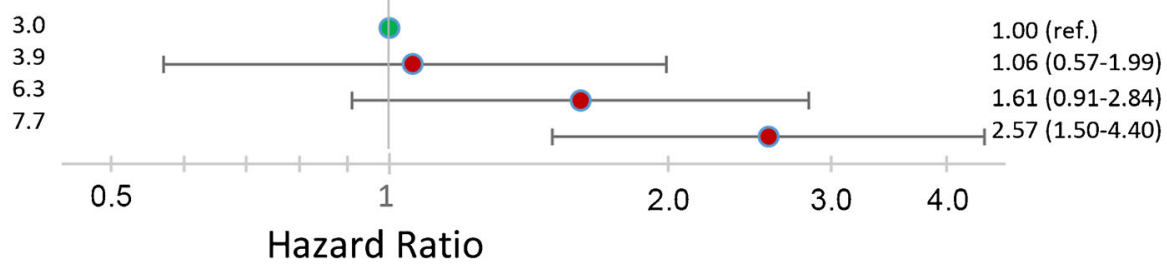

Hazard Ratio

Fig. 1 Adjusted risk for the primary composite outcome and all-cause mortality in CHARM-Preserved for each glycemia category

higher in patients with undiagnosed diabetes and prediabetes, compared with normoglycemic patients, $p<0.001$ for trend across dysglycemia categories for both HFpEF and HFrEF.

\section{Discussion}

We found that the prevalence of diagnosed diabetes was higher in patients with HFpEF (40\%) in CHARM than in those with $\mathrm{HFrEF}(35 \%)$. However, the novel data in the present report relate to the prevalence of undiagnosed diabetes and prediabetic dysglycemia, each of which were more common than a normal $\mathrm{HbA1c}$ in both HFpEF and HFrEF. Indeed, when undiagnosed and diagnosed diabetes were combined, a remarkable $62 \%$ of patients with each type of HF had diabetes. An additional fifth or so of patients had prediabetes, leaving just approximately one in six patients with a normal $\mathrm{HbAlc}$, an observation that was true for both HFrEF and HFpEF. In comparison 26\% had a normal $\mathrm{HbA} 1 \mathrm{c}$ and $49 \%$ had either diagnosed or undiagnosed diabetes in the Prospective Comparison of ARNI with ACEI to Determine Impact on Global Mortality and Morbidity in Heart Failure (PARADIGM-HF), and corresponding numbers were 30 and $41 \%$, respectively, in the Gruppo Italiano per lo Studio della Sopravvivenza nella Insufficienza Cardiaca-Heart Failure (GISSI-HF) trial [10]. One explanation for the higher prevalence in CHARM (irrespective of HF type) is that HbA1c was only measured in North American patients where the prevalence of diabetes is high, with the latest reports estimating that in the general population almost $10 \%$ have diagnosed or undiagnosed diabetes and another $34 \%$ of the population have prediabetes [11].

As was recently reported for patients with HFrEF, undiagnosed diabetes and prediabetic dysglycemia in patients with $\mathrm{HFpEF}$ (and HFrEF) in the present study were associated with worse outcomes than observed in patients with a normal

\section{$\mathrm{CV}$ death or $\mathrm{HFH}$}

No previous diagnosis of $D M$

Normoglycemia $(\mathrm{HbA} 1 \mathrm{c}<6.0)$

Prediabetes (HbA1c 6.0-6.4)

Diabetes ( $\mathrm{HbA1c}>6.4)$

Previous DM (any HbA1c)

All-cause mortality

No previous diagnosis of DM Normoglycemia (HbA1c $<6.0)$

Prediabetes (HbA1c 6.0-6.4)

Diabetes (HbA1c >6.4)

Previous DM (any HbA1c)
Number of events/

patients

$74 / 254$

$116 / 349$

$167 / 417$

$283 / 558$

\section{$43 / 254$}

$80 / 349$

$117 / 417$

$204 / 558$

\section{Incidence \\ rate per \\ $100 \mathrm{py}$}

11.4

13.3

17.0

22.9

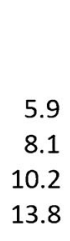

CHARM Alt + Added

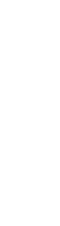

Adj. HR (95\% Cl)

1.00 (ref.)

1.01 (0.75-1.36)

$1.18(0.89-1.56)$

$1.65(1.27-2.15)$

1.00 (ref.)

$1.14(0.79-1.66)$

$1.28(0.90-1.83)$

$1.95(1.39-2.73)$

Hazard Ratio

Fig. 2 Adjusted risk for the primary composite outcome and all-cause mortality in CHARM-Alternative/Added for each glycemia category 
$\mathrm{HbA1c}$. Although this pattern was very similar to that reported in the PARADIGM-HF trial, no elevated risk was reported for patients with prediabetes in GISSI-HF. The difference in risk in the present study was not statistically significant, probably because of the smaller number of subjects with a HbAlc measurement in CHARM [7]. Although we found relative similar prevalences of dysglycemia in patients with HFpEF and HFrEF in the current study, the patients with these two phenotypes differed in respect of many of their baseline characteristics. Patients with HFrEF were more likely to have ischemic etiology, worse NYHA class and were less likely to be female. To some extent, the finding of a similarly high rate of dysglycemia in these two quite distinct phenotypes suggests that the syndrome of HF per se plays some role in the development of prediabetes and diabetes. Notably, insulin resistance is present in patients with idiopathic dilated cardiomyopathy as well as in those with ischemic cardiomyopathy, and it is greater in patients with coronary artery disease and HF than in patients with coronary artery disease without $\mathrm{HF}$ and is not correlated with ejection fraction [12-14]. These findings suggest that the high prevalence of dysglycemia in HF is not explained by recognized associations, e.g. with atherosclerosis and is related to HF per se, independently of ventricular function.

Like PARADIGM-HF, our study has the limitation of a single $\mathrm{HbA1c}$ value without a confirmatory measurement and the additional limitation of only including patients from North America where the prevalence of diabetes is higher than in other geographic regions, which may introduce a selection bias and impair applicability of our results to other regions of the world.

However, even with these limitations, our data confirm the remarkably high prevalence of dysglycemia in HFrEF and show that a similarly high prevalence is found in HFpEF (with only $16-18 \%$ of patients having a normal $\mathrm{HbA1c}$ ). In both types of $\mathrm{HF}$, dysglycemia is associated with a higher risk of adverse clinical outcomes, even before the diagnosis of diabetes. These findings raise questions about the potential value of screening (for undiagnosed diabetes) and treatment targeted at correcting dysglycemia in patients with both HF phenotypes [15].

Funding Information The CHARM programme was funded by AstraZeneca.

\section{Compliance with Ethical Standards}

Conflict of Interest Drs. Kristensen, Jhund and Lee report no relevant conflict of interests. All other authors have received honoraria as steering committee members of CHARM from AstraZeneca.

Ethical Approval All procedures performed in studies involving human participants were in accordance with the ethical standards of the institutional and/or national research committee and with the 1964 Helsinki declaration and its later amendments or comparable ethical standards. Informed consent was obtained from all individual participants included in the study.

Open Access This article is distributed under the terms of the Creative Commons Attribution 4.0 International License (http:// creativecommons.org/licenses/by/4.0/), which permits unrestricted use, distribution, and reproduction in any medium, provided you give appropriate credit to the original author(s) and the source, provide a link to the Creative Commons license, and indicate if changes were made.

\section{References}

1. Iribarren C, Karter AJ, Go AS, et al. Glycemic control and heart failure among adult patients with diabetes. Circulation. 2001;103: 2668-73.

2. He J, Ogden LG, Bazzano LA, Vupputuri S, Loria C, Whelton PK. Risk factors for congestive heart failure in US men and women: NHANES I epidemiologic follow-up study. Arch Intern Med. 2001;161:996-1002.

3. Held C, Gerstein HC, Yusuf S, et al. Glucose levels predict hospitalization for congestive heart failure in patients at high cardiovascular risk. Circulation. 2007;115:1371-5.

4. Targher G, Dauriz M, Laroche C, et al. In-hospital and 1-year mortality associated with diabetes in patients with acute heart failure: results from the ESC-HFA Heart Failure Long-Term Registry. Eur J Heart Fail. 2017;19:54-65.

5. MacDonald MR, Petrie MC, Varyani F, et al. Impact of diabetes on outcomes in patients with low and preserved ejection fraction heart failure: an analysis of the Candesartan in Heart failure: Assessment of Reduction in Mortality and morbidity (CHARM) programme. Eur Heart J. 2008;29:1377-85.

6. Gerstein HC, Swedberg K, Carlsson J, et al. The hemoglobin A1c level as a progressive risk factor for cardiovascular death, hospitalization for heart failure, or death in patients with chronic heart failure: an analysis of the Candesartan in Heart failure: Assessment of Reduction in Mortality and Morbidity (CHARM) program. Arch Intern Med. 2008;168:1699-704.

7. Kristensen SL, Preiss D, Jhund PS, et al. Risk related to prediabetes mellitus and diabetes mellitus in heart failure with reduced ejection fraction: insights from prospective comparison of ARNI with ACEI to determine impact on global mortality and morbidity in heart failure trial. Circ Heart Fail. 2016;9:e002560.

8. Pfeffer MA, Swedberg K, Granger CB, et al. Effects of candesartan on mortality and morbidity in patients with chronic heart failure: the CHARM-Overall programme. Lancet. 2003;362:759-66.

9. Authors/Task Force M, Rydén L, Grant PJ, et al. ESC guidelines on diabetes, pre-diabetes, and cardiovascular diseases developed in collaboration with the EASD: the task force on diabetes, pre-diabetes, and cardiovascular diseases of the European Society of Cardiology (ESC) and developed in collaboration with the European Association for the Study of Diabetes (EASD). Eur Heart J. 2013;34:3035-87.

10. Dauriz M, Targher G, Temporelli PL, et al. Prognostic impact of diabetes and prediabetes on survival outcomes in patients with chronic heart failure: a post-hoc analysis of the GISSI-HF (Gruppo Italiano per lo Studio della Sopravvivenza nella Insufficienza Cardiaca-Heart Failure) Trial. J Am Heart Assoc. 2017;6:e005156.

11. Centers for Disease Control and Prevention. National diabetes statistics report, 2017. U. S. Department of Health and Human Services; 2017.

12. Swan JW, Anker SD, Walton C, et al. Insulin resistance in chronic heart failure: relation to severity and etiology of heart failure. J Am Coll Cardiol. 1997;30:527-32.

13. Swan JW, Walton C, Godsland IF, Clark AL, Coats AJ, Oliver MF. Insulin resistance in chronic heart failure. Eur Heart J. 1994;15:1528-32.

14. Swan JW, Walton C, Godsland IF. Assessment of insulin sensitivity in man: a comparison of minimal model- and euglycaemic clamp-derived measures in health and heart failure. Clin Sci. 1994;86:317-22.

15. Zinman B, Wanner C, Lachin JM, et al. Empagliflozin, cardiovascular outcomes, and mortality in type 2 diabetes. N Engl J Med. 2015;373:2117-28. 